\title{
Ocorrência de internações por condições sensíveis à atenção primária (IC- SAP) no município de Campinas, São Paulo: associação com os atributos necessários a atenção primária
}

Occurrence of hospitalizations due to conditions sensitive to primary care in the city of Campinas, São Paulo: association with necessary attribute to primary health care

Márcia Regina Campos Costa da Fonseca

Laura Alejandra Matulevich Santana

Maria Eduarda Ribeiro Rojo

\section{RESUMO}

Introdução: As Internações por Condições Sensíveis à Atenção Primária (ICSAP) são um indicador indireto da efetividade do primeiro nível de atenção à saúde. Objetivo: Analisar a ocorrência de ICSAP e verificar a associação, nas unidades de saúde com maior e menor coeficiente ICSAP, com os atributos necessários à atenção primária à saúde (APS). Método: Estudo descritivo, sendo a fonte de dados das ICSAP, o Sistema de Informações Hospitalares-Campinas, 2015, e a fonte de dados referentes aos atributos das unidades de saúde, a entrevista aplicada à equipe de saúde, sendo um questionário avaliando a adequação da estrutura, baseado no Instrumento de Avaliação Externa do Programa Nacional de Melhoria do Acesso e da Qualidade da Atenção Básica (PMAQ) e o outro avaliando a adequação do processo de trabalho, através do Primary Care Assessment Tool PCATool-Brasil. Para as análises estatísticas foi utilizado o teste de Wilcoxon-MannWhitney, sendo $5 \%$ o nível de significância adotado nos testes. Resultados: As ICSAP corresponderam a 15,6\% do total de internações do município, sendo mais frequente entre os idosos $(48,6 \%)$. Não se observou significância estatística entre as ocorrências de ISCAP e os atributos da APS. Conclusão: Os achados deste estudo auxiliam na avaliação da atenção básica no Brasil.

Palavras-chave: Atenção Primária a Saúde. Avaliação de Serviços de Saúde; Indicadores de Qualidade em Assistência à Saúde. Hospitalização.

\section{ABSTRACT}

Introduction: Hospitalizations due to primary healthcare sensitive conditions are an indirect indicator of the effectiveness of the first level of health care. Objective: To analyze the occurrence of these hospitalizations verifying their association with aspects necessary for primary healthcare in health units with higher and lower occurrences. Methods: It is a descriptive study with data taken from Campinas' Hospital Information System (year 2015) and through two different surveys among managers and health teams. One of them being a questionnaire evaluating the adequacy of the structure based on the external evaluation instrument of the national program for improving access and quality of basic care, and the other evaluating the adequacy of the work process, through the Primary Care Assessment Tool (PCATool-Brazil). Statistical analyzes were performed using the Wilcoxon-Mann-Whitney test, with $5 \%$ as the level of significance adopted in the tests. Results: As occurrence of hospitalizations due to conditions sensitive to primary care corresponded $15.6 \%$ of the total hospitalizations of the municipality, being more frequent among the elderly (48.6\%). No statistical significance was observed between these occurrences and the primary health care attributes. Conclusion: The findings of this study helped in the evaluation of primary care in Brazil.

Keywords: Primary Health Care; Quality Indicators Heath Care; Health Services Evaluation; Hospitalization.

\section{INTRODUCTION}

A Política Nacional de Atenção Básica resulta da experiência acumulada de vários atores envolvidos historicamente com o desenvolvimento e a consolidação do Sistema Único de Saúde (SUS) e orienta-se pelos princípios da universalidade, da acessibilidade e da coordenação do cuidado, do vínculo e longitudinalidade, da integralidade, da responsabilização, da humanização, da equidade e da participação social [1].

A atenção básica ou internacionalmente designada de atenção primária à saúde (APS) é reconhecidamente

\footnotetext{
${ }^{1}$ Faculdade São Leopoldo Mandic, Curso de Medicina. Rua José Rocha Junqueira, 13, Swift, 13045-755, Campinas, SP, Brasil Correspondência para / Correspondence to: MRCC FONSECA. E-mail: <marcia.fonseca@slmandic.edu.br>. wh

Como citar este artigo / How to cite this article

Fonseca MRCC, Santana LAM, Rojo MRR. EOcorrência de internações por condições sensíveis à atenção primária (ICSAP) no município de Campinas, São Paulo: associação com os atributos necessários a atenção primária. InterAm J Med Health 2020;3:e202003002.
} 
um dos componentes-chave dos sistemas de saúde e seu impacto positivo na saúde é notório. Em países onde foi adotada como base para os sistemas de saúde, observa-se melhores indicadores de saúde, maior equidade, cuidados mais efetivos, maior investimento nas ações de promoção da saúde e prevenção de doenças, integralidade e resolutividade, com maior eficiência no fluxo dos usuários dentro do sistema [2].

O uso de indicadores para avaliar o acesso e a qualidade da APS foi inicialmente estudado nos Estados Unidos e posteriormente em outros países, sendo estes, valiosos para monitoramento e controle das ações desenvolvidas neste nível de atenção à saúde [3]. Um desses indicadores, denominado "ambulatory care sensitive conditions", foi desenvolvido por Billings e colaboradores (1990) e representa um conjunto de problemas de saúde para os quais a efetiva ação da APS diminuiria o risco de internações hospitalares [4].

No Brasil a discussão sobre esse indicador iniciouse no ano de 2005 e em 2008, através da Portaria 221, foi publicada a Lista Brasileira de Internações por Condições Sensíveis à Atenção Primária (ICSAP), composta por 19 grupos de condições [5].

O percentual de internações sensíveis a condições primárias no Brasil vem sofrendo diminuição (12,8\% em 2000; 9,1\% em 2015) [6] e estudos sugerem relação da tendência à diminuição com a Estratégia de Saúde da Família [7-11], adotada pelo Ministério da Saúde para a reorganização da atenção básica, e uma alternativa de ação para o alcance dos princípios e diretrizes do SUS [1].

As internações por ICSAP representa necessidade de análise por estar correlacionado com a deficiência na cobertura dos serviços e/ou baixa resolutividade da APS [3]. Através deste indicador é possível avaliar o desempenho de diferentes serviços de saúde, os efeitos de políticas de saúde e avaliar a resolutividade, qualidade e acessibilidade da atenção primária à saúde [3].

Diante do exposto este estudo teve como objetivo analisar a ocorrência de internações por condições sensíveis à atenção primária, no município de Campinas, São Paulo, e verificar a associação nas unidades básicas de saúde, com maior e menor coeficiente ICSAP, com os atributos necessários a atenção primária a saúde.

\section{METHODS}

Trata-se de um estudo descritivo e quantitativo, realizado em duas fases, sendo a primeira, retrospectiva, com a utilização de dados de ICSAP e a segunda, prospectiva, com a utilização de dados primários gerados por entrevista.

A primeira fase, constou de busca das Internações por Condições Sensíveis a Atenção Primária (ICSAP), ano de referência 2015, dados estes, extraídos do Sistema de Informações Hospitalares (SIH/SUS) disponibilizado pelo tabulador de dados (TABNET) da Secretaria Municipal de Saúde de Campinas [12]. As seguintes variáveis estavam disponíveis no sistema e foram levantadas: sexo e idade dos pacientes internados por ICSAP; número de ICSAP por distrito de saúde, bem como, número de ICSAP por unidade básica de saúde (UBS).

De posse destes dados foram realizados os cálculos dos coeficientes ICSAP por 100.000 habitantes, tendo no numerador o número de internações ICSAP e no denominador a população de abrangência dos distritos e das unidades básicas de saúde (UBS).

Após os cálculos dos coeficientes verificou-se as duas unidades de saúde, com menor e maior coeficiente ICSAP, para continuidade da segunda fase do estudo, realizada nos meses de dezembro de 2016 e janeiro de 2017. A unidade de saúde de menor coeficiente recebeu o nome fictício de "rosa" e a de maior coeficiente de "margarida".

Os dados coletados nas entrevistas tinham por objetivo avaliar a estrutura e processo de trabalho das unidades para avaliação de serviços de saúde, entendendo como "estrutura" os atributos materiais e organizacionais, características mais estáveis da assistência à saúde, e "processo" as atividades desenvolvidas entre profissionais de saúde e pacientes, relações que exercem respectiva influência na assistência [13].

O instrumento de pesquisa aplicado aos gestores, dados sobre a estrutura das unidades de saúde, foi baseado no instrumento de Avaliação Externa do Programa Nacional de Melhoria do Acesso e da Qualidade da Atenção Básica - PMAQ [14], sendo os itens investigados distribuídos em 11 categorias: os profissionais da equipe de atenção básica; sinalização; acessibilidade; reforma e ampliação; características estruturais e ambiência; tecnologia de informação; equipamentos e materiais; materiais impressos para a atenção à saúde; imunobiológicos e testes diagnósticos; insumos para a atenção à saúde e medicamentos. A cada item do questionário os gestores respondiam sim ou não. $\mathrm{O}$ instrumento de pesquisa aplicado às equipes de 
saúde, dados sobre o processo de trabalho das unidades, foi o "Primary Care Assessment Tool PCATool-Brasil" [15], sendo os escores investigados: (A) acesso de primeiro contato - acessibilidade (nove itens); (B) longitudinalidade (treze itens); (C) coordenação - integração dos cuidados (cinco itens); (D) coordenação - sistemas de informações (três itens); (E) integralidade - serviços disponíveis (vinte e dois itens); (F) integralidade - serviços prestados (quinze itens); $(G)$ orientação familiar (três itens) e $(H)$ orientação comunitária (seis itens).

Cada item do instrumento foi apresentado em uma escala de Likert: com certeza sim (4 pontos), provavelmente sim (3 pontos), provavelmente não (2 pontos), com certeza não (1 ponto), não sei ou não lembro (9 pontos), com inversão de valores para o escore (A9) acessibilidade - tempo de espera de mais de 30 minutos para os pacientes serem atendidos pelo médico ou enfermeiro sem contar triagem ou acolhimento, que quanto maior o valor atribuído, menor a orientação para atenção primária a saúde (APS), logo valor $4=1$; valor $2=3$; valor $2=3$ e valor $1=4$.

Posteriormente foi realizado a soma dos itens de cada escore e divisão pelo total de itens do mesmo. O escore essencial foi composto por $(A+B+C+D+E+F / 6)$ e 0 geral por $(\mathrm{A}+\mathrm{B}+\mathrm{C}+\mathrm{D}+\mathrm{E}+\mathrm{F}+\mathrm{G}+\mathrm{H} / 8)$.

$A$ análise de dados foi inicialmente descritiva e para os testes de associação entre os coeficientes ICSAP e atributos da APS foi utilizado o teste de Wilcoxon-MannWhitney, sendo $5 \%$ o nível de significância adotado nos testes estatísticos.

O estudo foi submetido e aprovado pelo Comitê de Ética da Faculdade São Leopoldo Mandic, parecer $n^{\circ}$ 1.829.770 e seguiu rigorosamente as recomendações da Resolução MS/CNS 510/16 e a Declaração de Helsinque.

\section{RESULTADOS E DISCUSSÃO}

Em 2015 foram registrados em Campinas 4.420 internações por condições sensíveis a atenção primária (ICSAP), o que correspondeu a $15,6 \%$ do total de internações do município, dado semelhante a município vizinho, Pedreira, com 15,9\% [16]. Estudos nacionais apontam variações nos indicadores de ICSAP, 11,54\% em Curitiba (PR) [17], 22,41\% no Espírito Santo [18], 36,6\% em Divinópolis (MG) [19] e 42,6\% em Bagé (RS) [20].

Das 4.420 internações por ICSAP praticamente não se observou diferença entre os sexos $(50,3 \%$ sexo masculino; 49,7\% sexo feminino), com pequena tendência de internações para o sexo masculino, diferente do apresentado em estudo realizado em Divinópolis (MG) com $56,1 \%$ das internações em mulheres [19].

Em relação à faixa etária $16,2 \%$ das ocorrências ICSAP foram registradas em jovens (0-19 anos), 35,2\% em adultos (20-59 anos) e 48,6\% em idosos ( $\geq 60$ anos), neste último, $32,4 \%$ das ICSAP eram no grupo de $\geq 80$ anos. Vários estudam relatam ser o grupo com mais idade o mais vulnerável a estas internações $[16,21,22]$.

O munícipio de Campinas se divide em cinco distritos de saúde sendo os Coeficientes ICSAP por 100.000 habitantes: distrito Norte $(308,3)$, distrito $\operatorname{Sul}(358,1)$, distrito Leste $(234,5)$, distrito Sudoeste $(467,2)$ e distrito Noroeste $(471,5)$. Em 353 registros o distrito de saúde foi ignorado.

Nestes distritos estão distribuídas 63 UBS. A unidade básica de saúde com menor coeficiente ICSAP (87,5/100.000 hab.), denominada de "rosa" pertence ao distrito Norte e a de maior coeficiente ICSAP $(820,2 / 100.000$ hab.), denominada de "margarida" pertence ao distrito Noroeste, e nestas foram aplicados os instrumentos de avaliação de estrutura e processo.

As respostas dos gestores das duas unidades de saúde avaliadas, quanto aos indicadores de estrutura, foram bem semelhantes. Diferença maior foi observada no item "tecnologia de informação" com mais respostas negativas para a UBS denominada "margarida" (tabela 1). A aplicação do instrumento de avaliação dos indicadores de processo ocorreu durante as reuniões de equipe, garantido assim, maior participação do grupo. Na UBS denominada "rosa" de um total de 36 funcionários, divididos em três equipes de saúde, participaram 31 $(86,1 \%)$, sendo assim distribuídos: equipe 1 (15 funcionários, participação de 13), equipe 2 (13 funcionários, participação de 11), equipe 3 (8 funcionários, participação de 6). Na UBS denominada "margarida" de um total de 42 funcionários, divididos em três equipes de saúde, participaram 34 (81,0\%), sendo assim distribuídos: equipe azul (15 funcionários, participação de 12), equipe lilás (11 funcionários, participação de 7), equipe amarela (16 funcionários, participação de 15).

A somatória dos itens de cada escore e divisão pelo total de itens do mesmo, guarda semelhança entre as unidades de saúde. O escore com menor pontuação foi acessibilidade, principalmente no que se refere aos itens: não funcionamento aos finais de semana; as unidades não estarem abertas em alguns dias da semana até as $20 \mathrm{hs}$ e 
Tabela 1. Distribuição das respostas dos gestores das duas unidades de saúde selecionadas segundo avaliação de estrutura. Campinas-SP. 2017.

\section{Profissionais da Equipe da $A B$}

Todas as ESF estão completas

Relação ESF/População adequada

Máximo 750 pessoas por ACS

Máximo 5 equipes por unidade

Território claramente definido

Presença de outros profissionais que compõem a equipe

Oferta número de consultas por habitante adequada (2 a 3)

\section{Sinalização}

Placa da fachada adequada

Sinalização de ambientes facilitando o acesso

Divulgação aos usuários (horários, ofertas serviços, escala funcionários)

Profissionais de saúde identificados com crachá

Identificação telefone ouvidoria

\section{Acessibilidade}

Entradas externas e internas, corredores adaptados para cadeiras de roda

Presença de cadeiras de rodas na unidade

Unidade dispõe de veículo para as ações externas (VD, busca ativa, etc.)

A disponibilidade de veículo atende as necessidades das equipes

\section{Reforma e Ampliação}

A unidade possui necessidade de reforma

Há necessidade de ampliação

Há espaço para ampliação

Reformas e ou ampliações ocorrem no tempo previsto

\section{Características estruturais e ambiência}

\section{Há sanitários para usuários, inclusive para os com deficiência física}

Há banheiros/vestuários funcionários

Há espaço físico para consultórios médicos, odontológicos e de enfermagem

Há área física para vacinas, curativos, farmácia, acolhimneto, etc

Ambientes dispõem de boa ventilação, iluminação, acústica, pisos e paredes laváveis, etc

\section{Tecnologia de informação}

Utilização de Sistema de informação para registro da informação (ex: SIAB, e-SUS/ SISAB)

Número adequado de equipamentos de informática (ex: computadores, impressoras, etc)

Número adequado de equipamentos eletrônicos (ex: datashow, TV, etc)

Acesso da equipe de saúde a internet

Banda larga disponível para realização das atividades

Número adequado de ambientes com computadores conectados à internet

\section{Equipamentos e materiais}

Equipamentos adequados e em quantidade suficientes

Materiais impressos para a atenção a saúde

Presença de caderneta de saúde da gestante, criança, vacinação, idoso

\section{Imunobiológicos e testes diagnósticos}

Presença de todos os imunobiológicos do calendário vacinal.

Presença de teste rápido de sífilis, HIV, gravidez

Disponibilidade constante e em quantidade adequada

\section{Insumos para a atenção a saúde}

Em quantidade adequada

\section{Medicamentos}

Presença de todos os medicamentos da cesta básica

Adequação oferta/demanda

Dispensação de medicamentos realizada por profissional qualificado para a função

\begin{tabular}{|c|c|}
\hline Rosa & Margarida \\
\hline Não & Não \\
\hline Não & Não \\
\hline Não & Não \\
\hline Sim & Sim \\
\hline Sim & Não \\
\hline Sim & Sim \\
\hline Não & Não \\
\hline Sim & Sim \\
\hline Sim & Sim \\
\hline Sim & Sim \\
\hline Não & Não \\
\hline Sim & Sim \\
\hline Sim & Não \\
\hline Sim & Sim \\
\hline Sim & Sim \\
\hline Não & Não \\
\hline Sim & Sim \\
\hline Sim & Sim \\
\hline Sim & Sim \\
\hline Não & Não \\
\hline Não & Sim \\
\hline Sim & Sim \\
\hline Sim & Sim \\
\hline Sim & Não \\
\hline Não & Não \\
\hline Sim & Sim \\
\hline Sim & Não \\
\hline Sim & Não \\
\hline Sim & Sim \\
\hline Sim & Sim \\
\hline Sim & Não \\
\hline Sim & Sim \\
\hline Sim & Sim \\
\hline Não & Sim \\
\hline Sim & Sim \\
\hline Não & Sim \\
\hline Não & Não \\
\hline Não & Não \\
\hline Não & Sim \\
\hline Sim & Sim \\
\hline
\end{tabular}


não disponibilizarem contato telefônico aos pacientes se estes necessitarem, quando estão fechadas (tabela 2).

Estudos apontam a diminuição das ICSAP em municípios com melhor cobertura da atenção básica e expansão da estratégia de saúde da família [7-11,23].

Em estudo de revisão com o objetivo de sintetizar as características da APS (estrutura, processo e desempenho dos serviços) associadas ao risco de hospitalização por ICSAP os autores destacam que a continuidade da atenção e a presença de equipe multidisciplinar mostraram-se associadas a menor probabilidade de hospitalização por ICSAP e concluem que, o seguimento dos princípios fundamentais da atenção primária, interferem neste indicador [24].

Neste estudo, a qualidade da atenção básica, medida através dos indicadores de estrutura e processo, não interferiu nos coeficientes de internações por condições sensíveis à atenção primária, uma vez que as duas unidades avaliadas, possuíam indicadores muito semelhantes, porém coeficientes de ICSAP muito díspares (tabela 2).

Tabela 2. Distribuição dos escores das unidades de saúde, segundo avalição de processo. Campinas-SP. 2017.

\begin{tabular}{|c|c|c|c|c|c|c|c|}
\hline \multirow[t]{3}{*}{ Itens avaliados } & \multicolumn{6}{|c|}{ Unidade de Saúde } & \multirow[t]{2}{*}{ p-valor } \\
\hline & \multicolumn{3}{|c|}{ Rosa } & \multicolumn{3}{|c|}{ Margarida } & \\
\hline & Equipe 1 & Equipe 2 & Equipe 3 & Amarela & Azul & Lilás & \\
\hline Acesso de primeiro contato - acessibilidade & 2,1 & 2,1 & 1,9 & 2,0 & 2,4 & 2,1 & 0,487 \\
\hline Longitudinalidade & 2,8 & 3,0 & 3,1 & 3,2 & 2,6 & 3,3 & 0,513 \\
\hline Coordenação - Integração dos Cuidados & 2,8 & 2,8 & 2,6 & 3,4 & 2,8 & 2,8 & 0,197 \\
\hline Coordenação - Sistemas de Informações & 3,3 & 3,3 & 3,3 & 4,0 & 3,0 & 4,0 & 0,480 \\
\hline Integralidade - Serviços Disponiveis & 3,3 & 3,4 & 3,3 & 3,7 & 3,4 & 3,5 & 0,072 \\
\hline Integralidade - Serviços Prestados & 3,4 & 3,4 & 3,5 & 3,5 & 3,1 & 3,3 & 0,369 \\
\hline Orientação Familiar & 3,7 & 3,7 & 4,0 & 4,0 & 3,8 & 4,0 & 0,239 \\
\hline Orientação Comunitária & 3,3 & 3,2 & 3,2 & 3,7 & 3,2 & 2,8 & 0,817 \\
\hline Escore Essencial & 3,0 & 3,0 & 3,0 & 3,3 & 2,9 & 3,2 & 0,487 \\
\hline Escore Geral & 3,1 & 3,1 & 3,1 & 3,4 & 3,0 & 3,2 & 0,487 \\
\hline
\end{tabular}

Fonte: Teste de Wilcoxon-Mann-Whitney.

Outros fatores além da qualificação da atenção primária, podem interferir nas ocorrências de ICSAP. Em estudo realizado no Espírito Santo, os autores não encontraram relação das ICSAP com a cobertura da ESF e do PACS, por outro lado, observou-se que o percentual de urbanização, de analfabetismo e de leitos SUS ofertados esteve positivamente associado ao risco de internações sensíveis [18]. Acesso à educação, renda, lazer, condições de moradia, ou seja, os determinantes sociais, podem influenciar a ocorrência dessas internações, sendo fatores exógenos à atenção básica [8].

Embora, não tenha sido objetivo desta pesquisa a análise da vulnerabilidade social das regiões onde estão inseridas as unidades de saúde com menor e maior coeficiente ICSAP, acredita-se que este fator possa interferir na ocorrência para este agravo à saúde. A falta desta análise é uma limitação deste estudo.

\section{CONCLUSÃO}

Neste estudo não se observou associação entre os coeficientes de internações sensíveis à atenção primária e os atributos de qualidade da atenção primária.

Ressalta-se que o indicador ICSAP deve ser utilizado para monitorar e avaliar a qualidade, o desempenho e o acesso à atenção primária e é ferramenta importante para o planejamento de políticas públicas e estratégias que visem a redução deste agravo à saúde.

\section{REFERENCES}

1. Brasil. Ministério da Saúde. Secretaria de Atenção Básica. Departamento de Atenção à Saúde. Política Nacional de Atenção Básica. Brasília: Ministério da Saúde; 2012.

2. Oliveira MAC, Pereira IC. Atributos essenciais da Atenção Primária e a Estratégia Saúde da Família. Rev Bras Enferm. 2013; 66:158-164.

3. Alfradique ME, Bonolo PF, Dourado I, Lima-Costa MF, Macinko J, Mendonça CS, et al. Internações por condições sensíveis à atenção primária: a construção da lista brasileira como ferramenta para medir o desempenho do sistema de saúde (Projeto ICSAP Brasil). Cad Saúde Pública. 2009;25(6):1337-1349.

4. Starfield B. Atenção Primária: equilíbrio entre a necessidade de saúde, serviços e tecnologias. Brasília: UNESCO; Ministério da Saúde; 2002.

5. Brasil. Ministério da Saúde. Portaria № 221, de 17 de abril de 2008. Lista Brasileira de Internações por Condições Sensíveis à Atenção Primária. Diário Oficial da República Federativa do Brasil, Brasília (DF). 2008.

6. Brasil. Ministério da Saúde. Fundação Oswaldo Cruz. PROADESS - Avaliação de desempenho do Sistema de Saúde Brasileiro: indicadores para monitoramento. Rio de Janeiro: Fundação Oswaldo Cruz; 2012. Disponível em:

http://www.proadess. icict.fiocruz.br/index.php?pag=res 1

7. Elias E, Magajewski F. A atenção primária à saúde no sul de Santa Catarina: uma análise das internações por condições sensíveis à atenção ambulatorial, no período de 1999 a 2004. Rev Bras Epidemiol. 2008;11(4): 633647. 
8. Rehem TCMSB, Ciosak SI,3 Egry EY. Internações por condições sensíveis à atenção primária no hospital geral de uma microrregião de saúde do município de São Paulo, Brasil. Texto Contexto Enferm. 2012; 21(3): 535-542.

9. Ceccon RF, Meneghel SN, Viecili PRN. Internações por condições sensíveis à atenção primária e ampliação da Saúde da Família no Brasil: um estudo ecológico. Rev Bras Epidemiol. 2014;17(4): 968-977.

10. Brasil VP, da Costa JSD. Hospitalizações por condições sensíveis à atenção primária em Florianópolis, Santa Catarina - estudo ecológico de 2001 a 2011. Epidemiol Serv Saúde. 2016;25(1):75-84.

11. da Costa JSD, Teixeira AMFB, Moraes M, Strauch ES, da Silveira DS, Carret MLV, et al. Hospitalizações por condições sensíveis à atenção primária em Pelotas: 1998 a 2012. Rev Bras Epidemiol. 2017;20(2):345-354.

12. Campinas. Secretaria Municipal de Saúde de Campinas. Coordenadoria de informação e Informática. Sistema de Informações Hospitalares (SIH-TabNet). Disponível em: http://tabnet.campinas.sp.gov.br/dh?aihs/ int.def

13. Donabedian A. The quality of care. How can it be assessed? Arch Pathol Lab Med. [periódico na internet]. 1997; 121(11): 1145-1150. Disponível em: http://post.

queensu.ca/ hh11/assets/applets/The_Quality_of_Car e How_Can_it_Be_Assessed_-_Donabedian.pdf

14. Brasil. Ministério da Saúde. Secretaria de Atenção Básica. Departamento de Atenção à Saúde. Programa Nacional de Melhoria do Acesso e da Qualidade da Atenção Básica (PMAQ). Instrumento de Avaliação Externa para as Equipes de Atenção Básica. Brasília: Ministério da Saúde; 2013.

15. Brasil. Ministério da Saúde. Secretaria de Atenção em Saúde. Departamento de Atenção Básica. Manual do instrumento de avaliação da atenção primária à saúde: primary care assessment tool pcatool. Brasília: Ministério da Saúde; 2010.

16. Torres RL, Rehem TCMSB, Egry EY, Ciosak SI. O panorama das internações por condições sensíveis à atenção primária em um distrito de São Paulo. Rev Esc Enferm USP. 2011;45(2):1661-1666.

17. Rehem TCMSB, Oliveira MRF, Amaral TCL, Ciosak SI, Egry EY. Internações por condições sensíveis à atenção primária em uma metrópole brasileira. Esc Enferm USP. 2013;47(4):884-890.
18. Pazó RG, Frauches DO, Molina MDCB, Cade NV. Modelagem hierárquica de determinantes a associados a internações por condições sensíveis à atenção primária no Espirito Santo, Brasil Cad Saúde Pública. 2014;30(9): 1891-1902.

19. Cardoso CS, Pádua CM, Rodrigues-Júnior $A A$, Guimarães DA, Carvalho SF, Valentin RF et al. Contribuição das internações por condições sensíveis à atenção primária no perfil das admissões pelo sistema público de saúde. Rev Panam Salud Publica. 2013; 34(4):227-234.

20. Nedel FB, Facchini LA, Martín-Mateo M, Vieira LAS, Thumé E. Programa Saúde da Família e condições sensíveis à atenção primária, Bagé (RS). Rev Saúde Pública. 2008;42(6):1041-1052.

21. Luciano TV, Dias JA. Internações por condições sensíveis a atenção primária e município da região Norte do Espírito Santo. Rev Bras Pesq Saúde. 2015;17(3): 23- 32.

22. Sousa NP, Rehem TCMSB, Santos WS, Santos CE. Internações sensíveis à atenção primária à saúde em hospital regional do Distrito Federal. Rev Bras Enferm. 2016;69(1):118-125.

23. Fernandes $\mathrm{VBL}$, Caldeira $\mathrm{AP}$, de Faria $\mathrm{AA}$, Rodrigues Neto JF. Internações sensíveis na atenção primária como indicador de avaliação da Estratégia Saúde da Família. Rev Saúde Pública. 2009;43(6):928-936.

24. Nedel FB, Facchini LA, Martín M, Navarro A. Características da atenção básica associadas ao risco de internar por condições sensíveis à atenção primária: revisão sistemática da literatura. Epidemiol Serv Saúde. 2010;19(1):61-75. 